\title{
CrimRxiv
}

\section{University of Missouri-St. Louis Comprehensive Safe Schools Initiative (UMSL CSSI)}

Finn-Aage Esbensen, Stephanie A. Wiley, Timothy McCuddy, Elaine Eggleston Doherty, Lee Ann Slocum, Terrance J. Taylor, Kyle Thomas, Matt Vogel

Published on: Dec 31, 2020

DOI: $10.21428 / c b 6 a b 371.8269139 b$

License: Creative Commons Attribution 4.0 International License (CC-BY 4.0). 
